\title{
Potential clinical application of IncRNAs in non-small cell lung cancer
}

This article was published in the following Dove Press journal:

OncoTargets and Therapy

\author{
Tong Lu' \\ Yuanyong Wang' \\ Di Chen ${ }^{2}$ \\ Jia Liu ${ }^{3}$ \\ Wenjie Jiao' \\ 'Department of Thoracic Surgery, \\ Affiliated Hospital of Qingdao \\ University, Qingdao, China; \\ ${ }^{2}$ Department of Gastroenterology, \\ Affiliated Hospital of Qingdao \\ University, Qingdao, China; ${ }^{3}$ School \\ of Pharmacy, Qingdao University, \\ Qingdao, China
}

\begin{abstract}
Lung cancer has been identified as one of the most prevalent and deadly tumors worldwide. In recent years, lncRNAs have been demonstrated to play a significant role in the development of lung cancer. Specifically, lncRNAs act as a regulator of cancer-critical genes, and they regulate the biological behavior of tumors at the transcriptional and posttranscriptional levels. Recent studies have shown that lncRNAs possess great potential in the treatment of non-small cell lung cancer patients because of their roles in diverse cellular processes, such as proliferation, metastasis, stem cell maintenance, and epithelial to mesenchymal transition, and they serve as signaling biomarkers. Compared to other invasive diagnostic methods, detection of lncRNAs may become a very useful noninvasive methodology. Moreover, lncRNAs can serve as potential therapeutic targets in non-small cell lung cancer due to their roles in regulating many signaling pathways associated with lung carcinoma. In this review, we discuss the roles and expression profile of 1 ncRNAs. We also discuss the promising application of 1 ncRNAs as predictors of clinical diagnosis, prognosis, and as potential therapeutic targets, aiming to demonstrate their practical value for clinical treatment.
\end{abstract}

Keywords: biomarker, lncRNA, diagnosis, prognosis, therapy, NSCLC

\section{Introduction}

Pulmonary malignancies are one of the most lethal cancers of humans. The prognosis of most lung cancer patients, whether they are suffering from non-small cell lung cancer (NSCLC) or small cell lung cancer, is quite poor with limited survival. In China, it is thought that there were 733,000 new cases of lung cancer and $>610,000$ related deaths in 2015, which ranked as the first for male patients and the second for female patients. ${ }^{1,2}$ Generally, the symptoms of early stage lung cancer are not obvious. Imaging modalities, such as computed tomography, and some serum tumor markers, such as carcinoembryonic antigen (CEA) and squamous cell carcinoma antigen (SCCA), are available for early diagnosis of NSCLC. However, traditional detection methods are inevitably limited by cumulative radiation damage and low sensitivity and specificity. ${ }^{3,4}$ New biomarkers with high sensitivity and specificity are needed for molecular diagnosis and prognosis; this can be done through deeper research of the molecular mechanisms of NSCLC.

The human genome has been identified by a number of computational and evolutionary analyses in the last few decades. Protein-coding genes, which account for only $1.5 \%$ of the genome, are well known for their vital functions in humans. 5,6 The remaining $98 \%$ of the human genome that does not encode proteins creates the so-called ncRNAs, which have received more attention recently for their potential functional roles. These ncRNAs are grouped into short ncRNAs and lncRNAs according 
to their size. ${ }^{7}$ The lncRNAs are a type of ncRNAs longer than 200 nucleotides, which are not translated into a protein, and regulate gene expression by multiple mechanisms. ${ }^{8}$ Many studies have shown lncRNAs to be implicated in several cellular biological functions, such as chromatin modification, gene expression regulation, cellular differentiation, and cell cycle progression. They can regulate mRNA splicing patterns and produce different splice variants, regulate downstream genetic transcription, modulate protein activity, serve as scaffolds for the assembly of multiple component complexes, be applied in transcriptional procession and regulate subcellular localization of protein. ${ }^{9,10}$ For instance, MEG3 has been identified to simulate the expression of GDF-15 by binding to factor p53. ${ }^{11}$ Similarly, lincRNA-p21 has been reported to be related to apoptosis, as it stimulates the inhibition of p53-dependent genes by activating p21 gene (CDKN1A). ${ }^{12}$ In addition, the lncRNA CCND1 was demonstrated to serve as an allosteric modulator of the RNA-binding protein TLS and inhibit transcription by bringing about repression of the cyclin D1 promoter. ${ }^{13}$ Consequently, considering the various functions that lncRNAs regulate, it is unsurprising that they are one of the primary features of some human cancers, including breast cancer, prostate cancer, lung cancer, colon cancer, and so on. These lncRNAs regulate tumor-critical genes in the development of cancers. In lung cancer, the reported lung cancer-associated lncRNAs include HOTAIR, H19, MALAT1, ANRIL, and GAS5, among others. Accordingly, lncRNAs' expression profiling is a reasonable approach to study tumor growth, invasion and metastasis, which might be feasible for diagnosis. ${ }^{14}$

A study of different targetable mutations, such as KARS, EGFR, HER2, MET, PI3KA, as well as ROS1 and ALK rearrangements, represents a critical junction for the therapy of lung carcinoma, paving the way to the era of personalized medicine. ${ }^{15,16}$ However, the 5-year overall survival (OS) of lung cancer of all stages together remains low at $15.9 \%{ }^{17}$ Further studies of lncRNAs have demonstrated them to be biomarkers or potential factors affecting cancer growth, invasion, metastasis, and recurrence. In this review, we aim to discuss the promising applications of lncRNAs as lung cancer biomarkers, thus bringing out novel findings about the noninvasive detection potentials for NSCLC diagnosis and prognosis as well as novel therapeutic targets for the attention of clinicians and researchers.

\section{LncRNAs related to NSCLC diagnosis}

Although many of the current markers for the diagnosis of NSCLC are proteins, ncRNAs are widespread in various body fluids and are relatively stable. ${ }^{18}$ Exploration of new ncRNAs for clinical diagnosis is a growing area of research. The stability of IncRNAs is similar to mRNA, while their tissue specificity is higher than mRNA, and they can also be detected in various body fluids such as blood, urine, and saliva; therefore, lncRNAs are suitable clinical indicators. ${ }^{19}$ Recently, numerous lncRNAs have been reported to act as tumor diagnosis biomarkers, including plasma HULC, which is a novel potential biomarker used for the confirmation of hepatocellular carcinoma. ${ }^{20}$ Additionally, PAC3 was used as a urinary biomarker for prostatic carcinoma diagnosis and prognosis.

Also, many abnormal lncRNAs have been identified in NSCLC. For example, MALAT1, on which nearly 100 studies have been published on its association with lung cancer, has been evaluated as a potential biomarker in body fluids. Research by Weber et al validated the expression of plasma lncRNA MALAT1 by distinguishing 45 NSCLC patients from healthy controls. These results indicated MALAT1 as a promising diagnostic biomarker due to its high specificity, stability, and minimal invasiveness (sensitivity, 56\%; specificity, 96\%; area under the receiver operating characteristic curve $=0.79) .{ }^{21}$ Also, serum exosomal MALAT1 may have clinical significance in the diagnosis of NSCLC. To study the expression of MALAT1 contained in exosome, Zhang et $\mathrm{al}^{22}$ selected 77 NSCLC patients and found the levels of MALAT1 from patients to be higher than healthy controls, with a test sensitivity of $60 \%$ and specificity of $81 \%(A U C=0.703)$. In their study, exosomal MALAT1 expression was positively associated with lymphatic node metastasis and TNM stage. However, other clinicopathologic features, including gender, age, and tumor diameter, showed no significant difference compared to exosomal MALAT1 levels.

As an early discovered lncRNA, accumulating evidence has indicated that GAS5 is downregulated in many cancers and functions as a tumor suppressor. ${ }^{23,24}$ For example, Liang et $\mathrm{al}^{25}$ designed a study to investigate the diagnostic value of GAS5 in blood samples, and they found that GAS5 was highly expressed in blood from NSCLC patients, compared with healthy controls, and the AUC reached 0.832 with a sensitivity of $82.2 \%$ and specificity of $72.7 \%$. In order to test this further, GAS5 and CEA were combined to identify the diagnostic efficiency, and the results showed that the combination yielded an AUC of 0.909 (95\% CI: 0.857-0.962; $P<0.001)$, which was significantly improved compared to GAS5 or CEA alone.

Similarly, the lncRNAs HIF1A-AS1 and XIST have been shown to play significant regulatory roles in tumor biology, with an elevated expression level in lung cancer. Tantai et $\mathrm{al}^{26}$ 
Table I IncRNAs related to NSCLC diagnosis

\begin{tabular}{|c|c|c|}
\hline IncRNA & Diagnostic efficacy & Reference \\
\hline MALATI & $\begin{array}{l}\text { Circulating MALATI, AUC }=0.79 \\
\text { Exosomal MALATI, AUC }=0.703\end{array}$ & $\begin{array}{l}21 \\
22\end{array}$ \\
\hline GAS5 & $\begin{array}{l}\text { Circulating GAS5, AUC }=0.832 \\
\text { Combination of GAS5 and CEA, AUC }=0.909\end{array}$ & 25 \\
\hline XIST and HIFIA-ASI & $\begin{array}{l}\text { Circulating XIST, AUC }=0.834 \\
\text { Circulating HIFIA-ASI, AUC }=0.876 \\
\text { Combination of XIST and HIFIA-ASI, AUC }=0.93 \mathrm{I}\end{array}$ & 26 \\
\hline SPRY4-ITI, ANRIL, and NEATI & $\begin{array}{l}\text { Circulating SPRY4-ITI, AUC }=0.603 \\
\text { Circulating ANRIL, AUC }=0.798 \\
\text { Circulating NEATI, AUC }=0.693 \\
\text { Combination of SPRY4-ITI, ANRIL, and NEATI; AUC }=0.876\end{array}$ & 27 \\
\hline SOX2OT and ANRIL & $\begin{array}{l}\text { Circulating SOX2OT, AUC }=0.745 \\
\text { Circulating ANRIL, AUC }=0.723 \\
\text { Combination of SOX2OT, ANRIL, CEA, CYFRA } 2 \mathrm{I}-\mathrm{I} \text {, and SCCA; AUC }=0.853\end{array}$ & 28 \\
\hline
\end{tabular}

Abbreviations: AUC, area under the receiver operating characteristic curve; NSCLC, non-small cell lung cancer; CEA, carcinoembryonic antigen.

explored the diagnostic values of these proteins in NSCLC. They found that compared to preoperative patients, patients with postsurgical intervention showed significantly lower expression levels of serum HIF1A-AS1 and XIST. The AUC reached 0.876 (95\% CI: 0.793-0.965; $P<0.001)$ for HIF1A-AS1 and 0.834 (95\% CI: $0.726-0.935 ; P<0.001)$ for XIST. Also, the combination of HIF1A-AS1 and XIST had higher diagnostic value, yielding an AUC of 0.931 (95\% CI: 0.869-0.990; $P<0.001)$.

Furthermore, in order to improve diagnostic efficiency, $\mathrm{Hu}$ et $\mathrm{al}^{27}$ selected 21 previously identified lncRNAs as potential targets for subsequent lncRNA detection. They eventually found that circulating NEAT1, ANRIL, and SPRY4-IT1 were significantly increased in plasma samples of NSCLC patients compared to control sets, and the combination of the three factors illustrated a higher power, with an AUC of $87.6 \%$ (sensitivity, 82.8\%; specificity, 92.3\%). These three factors might serve as promising biomarkers in the early identification of NSCLC due to their stability.

As tumor biomarkers, CEA, SCCA, and CYFRA21-1, among others, are not always able to work alone. Xie et $\mathrm{al}^{28}$ demonstrated that the combination of novel lncRNA biomarkers and traditional detection could have great potential in making NSCLC diagnosis. In their study, 14 lncRNAs were combined with three tumor biomarkers (SCCA, CEA, and CYFRA21-1) to predict NSCLC. Their results showed that SOX2OT and ANRIL might be potential biomarkers for such a diagnosis. In addition to establishing the role of lncRNAs in diagnosis, some studies have examined their use for the pathological classification of NSCLC. Zhao et $\mathrm{al}^{29}$ identified 72 lncRNAs with significant differences between lung squamous cell carcinoma and lung adenocarcinoma using gene microarray. White et $\mathrm{al}^{30}$ found 27 lung cancer-associated lncRNAs to be markers for the differential diagnosis of lung squamous cell carcinoma and lung adenocarcinoma.

Along with the aforementioned factors, many other novel lncRNAs, such as PVT1, ${ }^{31}$ CAR10, ${ }^{32}$ ZXF1, ${ }^{33}$ MetaLnc9, ${ }^{34}$ and PANDAR, ${ }^{35}$ among others, have been identified as playing multiple roles in NSCLC diagnosis and prognosis in recent years (Table 1). Although studies on these lncRNAs are not mature enough, accumulating evidence suggests that lncRNAs do have significant functions in epigenetic regulation, promote proliferation and cellular growth, and result in uncontrolled and progressive tumor growth and metastasis. Based on these findings, we can conclude that a single lncRNA by itself is not enough to be used as a clinical indicator in NSCLC due to its low sensitivity and specificity. However, combination of multiple lncRNAs for the diagnosis of NSCLC shows more reliable specificity and sensitivity. However, there is still no commonly recognized group of lncRNAs for NSCLC diagnosis.

\section{LncRNAs related to NSCLC therapy}

The most commonly used treatment for NSCLC currently includes surgical excision, chemotherapy, and chest radiotherapy. Surgical excision is especially available for early NSCLC patients. However, these therapies are limited to defeat advanced cancer due to their poor therapeutic efficacy. Novel approaches need to be explored and applied in the clinic before improving patient survival and quality of life. ${ }^{36}$ The lncRNAs have multiple roles in cancer progression and tumorigenesis and are widely expressed in lung carcinoma. They can be used as potential therapeutic targets in NSCLC due to their effects in the development and regulation of diverse molecular pathways correlated with 
gene expression. Significantly, dysregulated lncRNAs are reported to be associated with many treatments, including molecular-targeted therapy and chemotherapy, among others. For these reasons, lncRNAs can serve as new therapeutic targets for NSCLC by restoring the specificity and sensitivity of cancer cells to chemotherapeutic drugs and they may even cure the condition. ${ }^{37}$

\section{RNAi-mediated gene silencing therapy}

Generally, RNA interference technologies (shRNA, siRNA, and antisense oligonucleotides) represent the most promising approach for the selective inhibition of target lncRNAs, although in some cases, such a modality may not be available due to the introcellular localization or secondary structure. IncRNA-targeted RNAi has been proven to be effective using cell lines; however, stable conditions are needed to transport siRNA to the targets in vivo. Recently, numerous lncRNAs have been identified as potential therapeutic targets. For instance, HOTAIR silencing through RNAi has been shown to reduce invasiveness and viability in breast, pancreatic, as well as lung cancer, ${ }^{38}$ it also contributed to cisplatin resistance in lung adenocarcinoma cells via the downregulation of p21 expression. ${ }^{39}$ In addition, MALAT1 shRNA-mediated knockdown has been shown to significantly reduce cell invasiveness and migration in NSCLC. ${ }^{19}$

Remarkably, Cheng et al recently compared the pretreatment levels of UCA1 protein in EGFR-mutant NSCLC patients who had developed acquired resistance to EGFRtyrosine kinase inhibitors. UCA1 may play a significant role in acquiring resistance to EGFR-tyrosine kinase inhibitors. ${ }^{40}$ The delivery problems associated with siRNA and its offtarget effects may limit its further application, and inhibition of lncRNAs remains a challenge in vivo. ${ }^{41}$ However, several strategies have been developed to overcome this shortcoming, such as lipid-based nanoparticle delivery, ${ }^{42}$ conjugate-based delivery, ${ }^{43}$ and polymer-based delivery, ${ }^{44}$ and these approaches are available for potential molecular treatment.

\section{Antisense oligonucleotide (ASO)- based treatment}

ASOs are short single-stranded DNA that can induce lncRNA degradation via RNaseH; they can also be used for lncRNA silencing and regulation. Compared with siRNA, ASOs show fewer off-target effects and higher specificity. ${ }^{41}$ In some cases, ASOs were shown to target 1ncRNA MALAT1 and the inhibition of MALAT1 weakened malignant phenotypes via cycle arrest in cervical and lung cancer cells. ${ }^{45}$ As for uncovering the function of lncRNA MALAT1, Tony et al developed a MALAT1 knockout model in human lung tumor cells as a unique loss-of-function model. In their study, animals treated with MALAT1 ASO had significantly lower tumor volume and nodules in the lung compared to control ASO-treated animals. Thus, inhibition via MALAT1 ASOs prevented NSCLC metastasis, revealing a novel therapeutic approach for NSCLC patients' treatment. ${ }^{46}$

\section{Small molecule modulators of IncRNA-protein interactions}

Small molecule modulators mediate the regulation of lncRNAs by blocking the binding of lncRNAs to their interacting proteins. ${ }^{47}$ Small molecule inhibitors can be applied to bind lncRNA by mimicking or changing their secondary structure. Many chromatin-modifying enzymes are flexible, which can allow proteins to form stronger, more stable interactions and interact with specific partners. They can also form distinct structures when bound to different DNAs, RNAs, or proteins. ${ }^{48-51}$

Based on the above theory, small molecular modulators may develop novel therapeutic technology. For instance, targeting lncRNA-protein interactions would reduce offtarget effects and increase the specificity of compounds. Chromatin-modifying enzymes targeting lncRNA-protein interactions are more reliable compared to those targeting either lncRNAs or proteins alone. ${ }^{52}$ For this matter, HOTAIR interaction is inhibited along with LSD1 and PRC2 via small molecular modulators identified to reduce metastasis in breast carcinoma. ${ }^{53}$ However, there are still no reports about the lncRNA-related modulator in NSCLC. Therefore, in order to create novel small molecular drugs, lncRNA-protein interactions and pharmacological trends are needed to be explored and studied.

\section{Plasmid-mediated targeted therapy}

Another interesting approach for the treatment of cancer is plasmid-mediated targeted therapy. $\mathrm{H} 19$ has been identified to show high expression in breast cancer and lung cancer, among others. The plasmid BC-819, which carries the gene for the A subunit of diphtheria toxin, has been studied to utilize the tumor-specific expression of H19 lncRNA. ${ }^{54}$ BC-819 was also illustrated to play a role in the treatment of pancreatic, ovarian, and colon cancers and NSCLC. ${ }^{52}$

New biological technologies have emerged, such as RNAi, ASOs, and small molecular modulators. The potential use of lncRNAs for therapies is great, since they can provide 
a novel therapeutic strategy for patients with NSCLC and can be effective in restoring the normal expression levels of lncRNAs. However, several limitations still need to be addressed, such as selective tumor suppressor targets, the stability of lncRNAs in body fluids, and distribution of therapeutic lncRNAs. Due to the fact that many mechanisms of lncRNAs are still undiscovered, further studies are required to confirm these findings; however, it is unquestionable that lncRNAs can play an increasingly significant role in NSCLC treatment. The more we learn about lncRNAs, the higher the chance that an improved therapy can be developed.

\section{LncRNAs related to NSCLC prognosis}

The lncRNAs are closely related to NSCLC invasion and metastasis, as well as affect the prognosis of NSCLC patients. For example, MALAT1 has been investigated for its prognostic value in many studies. However, these studies were inevitably limited by their small sample sizes. Li et al ${ }^{55}$ performed an updated meta-analysis to summarize the potential value of MALAT1 as a biomarker for predicting survival in multiple human cancers, and they found raised MALAT1 could be regarded as an independent predictor for OS. In a study by Schmidt et al, it was found that high expression of MALAT1 were often associated with poor prognosis in squamous cell carcinoma of the lung. ${ }^{56}$ Furthermore, they showed that patients with low expression of MALAT1 had a better prognosis than those with high expression of MALAT1, and suggested that MALAT1 could serve as a biomarker for the prognosis of NSCLC patients.

Besides MALAT1, Zhang et $\mathrm{al}^{57}$ investigated the lncRNA H19, including its biological roles and clinical significance. They stated that elevated H19 predicted a poor prognosis and was an independent prognostic factor for OS. Also, Nakagawa et al found that the expression of HOTAIR in early brain metastases of NSCLC patients was much higher than in primary lesions, and suggested that HOTAIR could be used as a predictor of early tumor cell metastasis in NSCLC patients. HOTAIR could also promote tumor cell migration in vitro. ${ }^{58}$ Liu et $\mathrm{al}^{39}$ found that overexpression of HOTAIR presented with lymph node metastasis, advanced stage of disease, and predicted poor prognosis. Compared with the high expression levels of HOTAIR patients, the lower expression group experienced a longer OS.

Accordingly, Qiu et al studied the lncRNA CCAT2 expression levels in lung cancer and found that overexpression of CCAT2 was significantly associated with lung adenocarcinoma $(P=0.033)$, but not with squamous cell cancer.
They also demonstrated that elevated CCAT2 expression promoted the invasion and proliferation of lung adenocarcinoma cells, indicating a poor prognosis. ${ }^{59}$ Luo et al found that patients with high CARLo-5 expression levels presented poorer prognosis, whereas patients with lower expression of CARLo-5 experienced a longer OS. ${ }^{60}$ Besides the above, the lncRNAs ZXF1, ${ }^{33}$ BANCR, ${ }^{61}$ and PVT $1{ }^{62}$ were also investigated in various studies and proved to be potential prognostic biomarkers for NSCLC.

The combination of multiple lncRNA expressions was investigated for use in the prognosis of NSCLC patients. In order to determine the prognostic value of multiple lncRNAs in NSCLC patients, Zhang et al studied the correlation between clinical outcomes and lncRNA expression. According to the median gene expression, patients were divided into different groups. The researchers were able to demonstrate that high expression of H19, MALAT1, and HOTAIR and low expression of TUG1 and PANDA were predictors of poor prognosis. Different risk groups are managed according to the expression of lncRNAs. In the results, the disease-free survival time curves differed significantly among the low-risk, moderate-risk, and high-risk groups. ${ }^{63}$ There are also many studies analyzing the correlation between IncRNAs and their clinical significance in silico. The association between the expression of MEG3 and prognosis in NSCLC found using the Gene Expression Omnibus database showed that low expression of MEG3 experienced an unfavorable prognosis in NSCLC (Table 2). ${ }^{64}$

Although many lncRNAs, such as MALAT1, HOTAIR, H19, CCAT2, and so on, are associated with NSCLC prognosis, there is no clinical study showing that lncRNAs can be used in a practical judgment of the prognosis of NSCLC. The prognostic value of lncRNAs thus remains limited to preclinical research and has no clinical application value currently.

\section{Discussion and perspectives}

In this review, we highlighted the clinical application of lncRNAs, especially their potential application for diagnosis, therapy, and prognosis in NSCLC. In sum, lncRNAs are actively involved in multiple signaling pathways and have been regarded as important regulators of diverse cellular processes. Growing evidence suggests that they participate in various cellular processes, including cell growth, migration, stem cell maintenance, and apoptosis. ${ }^{65}$ In the clinic, imaging diagnosis and hematological examinations are the most commonly used methods to detect NSCLC. However, the efficiency of traditional methods is unsatisfactory. Since the identification 
Table 2 IncRNAs related to NSCLC prognosis

\begin{tabular}{|c|c|c|c|}
\hline IncRNA & Function in NSCLC & Function & Reference \\
\hline MALATI & Promotes cell proliferation, migration, and invasion & Poor prognosis & 55,56 \\
\hline $\mathrm{HI9}$ & Promotes cell growth & Poor prognosis & 57 \\
\hline HOTAIR & Promotes cell proliferation, invasion, and metastasis & Poor prognosis & 38,58 \\
\hline ССАТ2 & Promotes cell proliferation and invasion & Poor prognosis & 59 \\
\hline CARLo-5 & Promotes cell proliferation, migration, and invasion & Poor prognosis & 60 \\
\hline BANCR & Suppresses cell proliferation, induces apoptosis & Poor prognosis & 61 \\
\hline PVTI & Promotes cell proliferation, migration, and invasion & Poor prognosis & 62 \\
\hline ZXFI & Promotes cell invasion and metastasis & Poor prognosis & 33 \\
\hline TUGI & Suppresses cell proliferation & Poor prognosis & 63 \\
\hline PANDAR & Represses cell proliferation & Poor prognosis & \\
\hline MEG3 & Promotes cell invasion and metastasis & Poor prognosis & 64 \\
\hline
\end{tabular}

Abbreviation: NSCLC, non-small cell lung cancer.

of CEA in 1965, various studies have demonstrated its role in the follow-up of NSCLC patients. ${ }^{66}$ Approximately $70 \%$ of NSCLC patients have CEA that is overexpressed in advanced stages ${ }^{67} \mathrm{CEA}$ is useful for monitoring disease and detecting disease recurrence, metastasis, and prognosis. ${ }^{68,69}$ However, the use of CEA is clinically controversial as a predictive and prognostic marker for lung cancer patients as its specificity is higher but its sensitivity is lower than other biomarkers in the diagnosis of NSCLC patients. ${ }^{69}$

For a long time, chemotherapy has been one of the major therapeutic modalities in oncology. Classical chemotherapy can block cellular proliferation, mainly by interrupting mitotic division and DNA synthesis. However, positive clinical outcomes using this approach have remained limited. Accordingly, with the significant advances in the genetic study of lncRNAs, novel methods should be explored for diagnosis, therapy, and prognosis, but potential clinical applications of lncRNAs are significant.

For example, lncRNAs are aberrantly expressed in cancers and exhibit stability in tissues and body fluids. Another advantage is that targeting lncRNAs have no side effect on normal tissues. Therefore, selectively targeting dysregulated lncRNAs could provide a new therapeutic strategy for NSCLC treatment. Over the last decade, many lncRNAs have served as diagnostic and prognostic biomarkers in their regulation of lung cancer. Lung tissue-specific genes should be explored to guide clinical practice. As reviewed in this paper, lncRNAs represent potentially noninvasive biomarkers in NSCLC diagnosis and prognosis.

Moreover, lncRNAs might serve as predictive biomarkers by representing the sensitivity of targeted oncology therapies. Based on the study of IncRNA biology, lncRNA-based therapies, such as ASOs, hold promising potential for developing effective personalized anticancer treatment strategies in lung cancer patients as well as for overcoming chemoresistance. However, we have to acknowledge that studies on lncRNAs still do not tell the whole mechanistic story. Even though some lncRNAs have been identified, there are still difficulties in applying them to the clinic. We must consider the standardization of sample preparation protocols, stability of lncRNA vectors in body fluids, developing a delivery system for adequate distribution and dosing lncRNA, and extraction methods, among other issues.

Researchers are paying more attention to the novel molecular biomarkers outlined above and are translating these achievements for use in the clinic. As biomedical research progresses, IncRNAs are believed to have the potential to have considerable clinical value in oncology.

\section{Acknowledgment}

The Department of Thoracic Surgery, Affiliated Hospital of Qingdao University supported this study.

\section{Disclosure}

The authors report no conflicts of interest in this work.

\section{References}

1. Mao Y, Yang D, He J, Krasna MJ, Ding Y, Jie H. Epidemiology of lung cancer. Surg Oncol Clin N Am. 2016;25(3):439-445.

2. Chen W, Zheng R, Baade PD, et al. Cancer statistics in China, 2015. CA Cancer J Clin. 2016;66(2):115-132.

3. I H, Cho JY. Lung cancer biomarkers. Adv Clin Chem. 2015;72(9):107.

4. Welch HG. Cancer screening, overdiagnosis, and regulatory capture. JAMA Intern Med. 2017;177(7):915-916.

5. ENCODE Project Consortium; Birney E, Stamatoyannopoulos JA, Dutta A, et al. Identification and analysis of functional elements in $1 \%$ of the human genome by the ENCODE pilot project. Nature. 2007; 447(7146):799-816.

6. Lander E, Linton L, Birren B, et al. Initial sequencing and analysis of the human genome [see comment] [erratum appears in Nature 2001]. Nature. 2001;412(6846):565.

7. St Laurent G, Wahlestedt C, Kapranov P. The landscape of long noncoding RNA classification. Trends Genet. 2015;31(5):239-251. 
8. Spizzo R, Almeida MI, Colombatti A, Calin GA. Long non-coding RNAs and cancer: a new frontier of translational research? Oncogene. 2012;31(43):4577-4587.

9. Wilusz JE, Sunwoo H, Spector DL. Long noncoding RNAs: functional surprises from the RNA world. Genes Dev. 2009;23(13):1494-1504.

10. Li G, Zhang H, Wan X, et al. Long noncoding RNA plays a key role in metastasis and prognosis of hepatocellular carcinoma. Biomed Res Int. 2014;2014(5147):780521.

11. Benetatos L, Vartholomatos G, Hatzimichael E. MEG3 imprinted gene contribution in tumorigenesis. Int J Cancer. 2011;129(4):773-779.

12. Huarte M, Guttman M, Feldser D, et al. A large intergenic noncoding RNA induced by p53 mediates global gene repression in the p53 response. Cell. 2010;142(3):409-419.

13. Wang X, Arai S, Song X, et al. Induced ncRNAs allosterically modify RNA-binding proteins in cis to inhibit transcription. Nature. 2008; 454(7200):126-130.

14. Tano K, Akimitsu N. Long non-coding RNAs in cancer progression. Front Genet. 2012;3:219.

15. Sharma SV, Bell DW, Settleman J, Haber DA. Epidermal growth factor receptor mutations in lung cancer. Nat Rev Cancer. 2007;7(3): 169-181.

16. Bergethon K, Shaw AT, Ou SH, et al. ROS1 rearrangements define a unique molecular class of lung cancers. J Clin Oncol. 2012;30(8): 863-870.

17. Ettinger DS, Akerley W, Borghaei H, et al. Non-small cell lung cancer, version 2.2013. J Natl Compr Canc Netw. 2013;11(6):645-653.

18. Cortez MA, Bueso-Ramos C, Ferdin J, Lopez-Berestein G, Sood AK, Calin GA. MicroRNAs in body fluids - the mix of hormones and biomarkers. Nat Rev Clin Oncol. 2011;8(8):467-477.

19. Qi P, Du X. The long non-coding RNAs, a new cancer diagnostic and therapeutic gold mine. Mod Pathol. 2013;26(2):155-165.

20. Leyten GH, Hessels D, Jannink SA, et al. Prospective multicentre evaluation of PCA3 and TMPRSS2-ERG gene fusions as diagnostic and prognostic urinary biomarkers for prostate cancer. Eur Urol. 2014;65(3):534-542.

21. Weber DG, Johnen G, Casjens S, et al. Evaluation of long noncoding RNA MALAT1 as a candidate blood-based biomarker for the diagnosis of non-small cell lung cancer. BMC Res Notes. 2013;6(1):518.

22. Zhang $R$, Xia $Y$, Wang Z, et al. Serum long non coding RNA MALAT-1 protected by exosomes is up-regulated and promotes cell proliferation and migration in non-small cell lung cancer. Biochem Biophys Res Commun. 2017;490(2):406-414.

23. Pickard MR, Mourtada-Maarabouni M, Williams GT. Long non-coding RNA GAS5 regulates apoptosis in prostate cancer cell lines. Biochim Biophys Acta. 2013;1832(10):1613-1623.

24. Sun M, Jin FY, Xia R, et al. Decreased expression of long noncoding RNA GAS5 indicates a poor prognosis and promotes cell proliferation in gastric cancer. BMC Cancer. 2014;14(1):319.

25. Liang W, Lv T, Shi X, et al. Circulating long noncoding RNA GAS5 is a novel biomarker for the diagnosis of nonsmall cell lung cancer. Medicine. 2016;95(37):e4608.

26. Tantai J, Hu D, Yang Y, Geng J. Combined identification of long non-coding RNA XIST and HIF1A-AS1 in serum as an effective screening for non-small cell lung cancer. Int J Clin Exp Pathol. 2015; 8(7):7887

27. Hu X, Bao J, Wang Z, et al. The plasma lncRNA acting as fingerprint in non-small-cell lung cancer. Tumour Biol. 2016;37(3):3497-3504.

28. Xie Y, Zhang Y, Du L, et al. Circulating long noncoding RNA act as potential novel biomarkers for diagnosis and prognosis of non-small cell lung cancer. Mol Oncol. 2018;12(5):648-658.

29. Zhao W, Luo J, Jiao S. Comprehensive characterization of cancer subtype associated long non-coding RNAs and their clinical implications. Sci Rep. 2014;4(10):6591.

30. White NM, Cabanski CR, Silva-Fisher JM, Dang HX, Govindan R, Maher CA. Transcriptome sequencing reveals altered long intergenic non-coding RNAs in lung cancer. Genome Biol. 2014;15(8):429.
31. Wu D, Li Y, Zhang H, Hu X. Knockdown of Lncrna PVT1 enhances radiosensitivity in non-small cell lung cancer by sponging Mir-195. Cell Physiol Biochem. 2017;42(6):2453-2466.

32. Wei MM, Zhou YC, Wen ZS, et al. Long non-coding RNA stabilizes the Y-box-binding protein 1 and regulates the epidermal growth factor receptor to promote lung carcinogenesis. Oncotarget. 2016;7(37): 59556-59571.

33. Zhang L, Zhou XF, Pan GF, Zhao JP. Enhanced expression of long non-coding RNA ZXF1 promoted the invasion and metastasis in lung adenocarcinoma. Biomed Pharmacother. 2014;68(4):401-407.

34. Yu T, Zhao Y, Hu Z, et al. MetaLnc9 facilitates lung cancer metastasis via a PGK1-activated AKT/mTOR pathway. Cancer Res. 2017;77(21): 5782-5794.

35. Han L, Zhang EB, Yin DD, et al. Low expression of long noncoding RNA PANDAR predicts a poor prognosis of non-small cell lung cancer and affects cell apoptosis by regulating Bcl-2. Cell Death Dis. 2015;6(2):e1665.

36. Sang H, Liu H, Xiong P, Zhu M. Long non-coding RNA functions in lung cancer. Tumour Biol. 2015;36(6):4027-4037.

37. Ricciuti B, Mencaroni C, Paglialunga L, et al. Long noncoding RNAs: new insights into non-small cell lung cancer biology, diagnosis and therapy. Med Oncol. 2016;33(2):18.

38. Yao Y, Li J, Wang L. Large intervening non-coding RNA HOTAIR is an indicator of poor prognosis and a therapeutic target in human cancers. Int J Mol Sci. 2014;15(10):18985-18999.

39. Liu Z, Sun M, Lu K, et al. The long noncoding RNA HOTAIR contributes to cisplatin resistance of human lung adenocarcinoma cells via downregualtion of $\mathrm{p} 21$ (WAF1/CIP1) expression. PLoS One. 2013; 8(10): e77293.

40. Cheng N, Cai W, Ren S, et al. Long non-coding RNA UCA1 induces non-T790M acquired resistance to EGFR-TKIs by activating the AKT/mTOR pathway in EGFR-mutant non-small cell lung cancer. Oncotarget. 2015;6(27):23582-23593.

41. Li CH, Chen Y. Targeting long non-coding RNAs in cancers: progress and prospects. Int J Biochem Cell Biol. 2013;45(8):1895-1910.

42. Whitehead KA, Langer R, Anderson DG. Knocking down barriers: advances in siRNA delivery. Nat Rev Drug Discov. 2009;8(2):129-138.

43. Lee HY, Mohammed KA, Kaye F, et al. Targeted delivery of let-7a microRNA encapsulated ephrin-A1 conjugated liposomal nanoparticles inhibit tumor growth in lung cancer. Int J Nanomedicine. 2013;8(Issue 1): 4481-4493.

44. Thomas M, Lu JJ, Ge Q, Zhang C, Chen J, Klibanov AM. Full deacylation of polyethylenimine dramatically boosts its gene delivery efficiency and specificity to mouse lung. Proc Natl Acad Sci US A. 2005 102(16):5679-5684.

45. Tripathi V, Shen Z, Chakraborty A, et al. Long noncoding RNA MALAT1 controls cell cycle progression by regulating the expression of oncogenic transcription factor B-MYB. PLoS Genet. 2013;9(3): e1003368

46. Gutschner T, Hämmerle M, Eissmann M, et al. The noncoding RNA MALAT1 is a critical regulator of the metastasis phenotype of lung cancer cells. Cancer Res. 2013;73(3):1180-1189.

47. Ayers D. Long non-coding RNAs: novel emergent biomarkers for cancer diagnostics. J Cancer Res Treat. 2013;1(2):31-35.

48. Turoverov KK, Kuznetsova IM, Uversky VN. The protein kingdom extended: ordered and intrinsically disordered proteins, their folding, supramolecular complex formation, and aggregation. Prog Biophys Mol Biol. 2010;102(2-3):73-84.

49. Uversky VN. The mysterious unfoldome: structureless, underappreciated, yet vital part of any given proteome. J Biomed Biotechnol. 2010;2010(1110-7243):1-14.

50. Uversky VN, Dunker AK. Understanding protein non-folding. Biochim Biophys Acta. 2010;1804(6):1231-1264.

51. Castello A, Fischer B, Eichelbaum K, et al. Insights into RNA biology from an atlas of mammalian mRNA-binding proteins. Cell. 2012; 149(6):1393-1406. 
52. Fatemi RP, Velmeshev D, Faghihi MA. De-repressing lncRNA-targeted genes to upregulate gene expression: focus on small molecule therapeutics. Mol Ther Nucleic Acids. 2014;3(11):e196.

53. Wu L, Murat P, Matak-Vinkovic D, Murrell A, Balasubramanian S. Binding interactions between long noncoding RNA HOTAIR and PRC2 proteins. Biochemistry. 2013;52(52):9519-9527.

54. Mizrahi A, Czerniak A, Levy T, et al. Development of targeted therapy for ovarian cancer mediated by a plasmid expressing diphtheria toxin under the control of H19 regulatory sequences. J Transl Med. 2009;7(1):69.

55. Li Y, Yang Z, Wan X, et al. Clinical prognostic value of metastasisassociated lung adenocarcinoma transcript 1 in various human cancers: an updated meta-analysis. Int J Biol Markers. 2016;31(2):e173-e182.

56. Schmidt LH, Spieker T, Koschmieder S. The long noncoding MALAT-1 RNA indicates a poor prognosis in non-small cell lung cancer and induces migration and tumor growth. J Thorac Oncol. 2011;6(12): 1984-1992.

57. Zhang E, Li W, Yin D, et al. Erratum to: c-Myc-regulated long non-coding RNA H19 indicates a poor prognosis and affects cell proliferation in non-small-cell lung cancer. Tumour Biol. 2016;37(4):5653.

58. Nakagawa T, Endo H, Yokoyama M, et al. Large noncoding RNA HOTAIR enhances aggressive biological behavior and is associated with short disease-free survival in human non-small cell lung cancer. Biochem Biophys Res Commun. 2013;436(2):319-324.

59. Qiu M, Xu Y, Yang X, et al. CCAT2 is a lung adenocarcinoma-specific long non-coding RNA and promotes invasion of non-small cell lung cancer. Tumour Biol. 2014;35(6):5375-5380.

60. Luo J, Tang L, Zhang J, et al. Long non-coding RNA CARLo-5 is a negative prognostic factor and exhibits tumor pro-oncogenic activity in non-small cell lung cancer. Tumour Biol. 2014;35(11):11541-11549.
61. Sun M, Liu XH, Wang KM, et al. Downregulation of BRAF activated non-coding RNA is associated with poor prognosis for non-small cell lung cancer and promotes metastasis by affecting epithelial-mesenchymal transition. Mol Cancer. 2014;13(1):68.

62. Cui D, Yu CH, Liu M, Xia QQ, Zhang YF, Jiang WL. Long non-coding RNA PVT1 as a novel biomarker for diagnosis and prognosis of nonsmall cell lung cancer. Tumour Biol. 2016;37(3):4127-4134.

63. Zhang CG, Yin DD, Sun SY, Han L. The use of lncRNA analysis for stratification management of prognostic risk in patients with NSCLC. Eur Rev Med Pharmacol Sci. 2017;21(1):115.

64. Zhang Z, Liu T, Wang K, et al. Down-regulation of long non-coding RNA MEG3 indicates an unfavorable prognosis in non-small cell lung cancer: evidence from the GEO database. Gene. 2017;630:49-58.

65. Kopp F, Mendell JT. Functional classification and experimental dissection of long noncoding RNAs. Cell. 2018;172(3):393-407.

66. Moro D, Villemain D, Vuillez JP, Delord CA, Brambilla C. CEA, CYFRA21-1 and SCC in non-small cell lung cancer. Lung Cancer. 1995; 13(2):169-176.

67. Robbins PF, Eggensperger D, Qi CF, Schlom J. Definition of the expression of the human carcinoembryonic antigen and non-specific crossreacting antigen in human breast and lung carcinomas. Int J Cancer. 1993;53(6):892-897.

68. Lee DS, Kim SJ, Kang JH, et al. Serum carcinoembryonic antigen levels and the risk of whole-body metastatic potential in advanced non-small cell lung cancer. J Cancer. 2014;5(8):663-669.

69. Grunnet M, Sorensen JB. Carcinoembryonic antigen (CEA) as tumor marker in lung cancer. Lung Cancer. 2012;76(2):138-143.
OncoTargets and Therapy

\section{Publish your work in this journal}

OncoTargets and Therapy is an international, peer-reviewed, open access journal focusing on the pathological basis of all cancers, potential targets for therapy and treatment protocols employed to improve the management of cancer patients. The journal also focuses on the impact of management programs and new therapeutic agents and protocols on

\section{Dovepress}

patient perspectives such as quality of life, adherence and satisfaction The manuscript management system is completely online and includes a very quick and fair peer-review system, which is all easy to use. Visit http://www.dovepress.com/testimonials.php to read real quotes from published authors. 